Pacific Journal of Mathematics

FUNCTION ALGEBRAS OVER VALUED FIELDS 


\title{
FUNCTION ALGEBRAS OVER VALUED FIELDS
}

\author{
G. Bachman, E. Beckenstein and L. Narici
}

In this paper we consider primarily algebras $F(T)$ of continuous funtions taking a topological space $T$ into a complete nonarchimedean nontrivially valued field $F$. Some general properties of function algebras and topological algebras over valued fields are developed in $\S \S 1$ and 2 . Some principal results (Theorems 6 and 7) are analogs of theorems of Nachbin and Shirota, and Warner: Essentially that $F(T)$ with compact-open topology is $F$-barreled iff unbounded functions exist on closed noncompact subsets of $T$; and that full Fréchet algebras are realizable as function algebras $F(\mathscr{L C})$ where $\mathscr{C}$ denotes the space of nontrivial continuous homomorphisms of the algebra.

Nachbin and Shirota's well-known result provides a necessary and sufficient condition for an algebra of realvalued continuous functions on a topological space to be barreled when it carries the compactopen topology. To develop an analog of Nachbin's theorem for $F$-valued functions, it is necessary to bypass the heavily real-number-oriented machinery on which his proof depends. We accomplish this in part by developing an ordering of the elements of a discretely valued field (Sec. 3, Def. 2) which serves to take the place of the usual ordering of the reals. We also consider a notion of "support" of a continuous $F$-valued linear functional on $F(T)$ (Sec. 3, Def. 3). The support notion is developed without measure theory or representation theorems for continuous linear functionals.

The results of the paper depend heavily on theorems proved by Ellis ([3]), Kaplansky ([7], [8]), and van Tiel ([14]), as well as the proofs of the major theorems as originally presented by Nachbin ([10]) and Warner ([15]) which provided the ideas for this line of approach.

Throughout the paper "algebra" (denoted by $X$ or $Y$ ) includes the presence of an identity and commutativity. The underlying field $F$ is assumed to be a complete nonarchimedean rank one nontrivially valued field. Unless otherwise stated, $T$ denotes a 0 -dimensional (a base for the topology consisting of closed and open sets exists) Hausdorff topological space and $F(T)$ the algebra of continuous functions from $T$ into $F$ with pointwise operations. The terms Banach space or Banach algebra are used throughout in the sense of [12].

1. Topological algebras over valued fields. In this section we discuss some basic properties of topological algebras over fields with valuation. We assume throughout that the underlying field $F$ is a 
complete nonarchimedean rank one nontrivially valued field.

Definition 1. A topological algebra $X$ over $F$ is nonarchimedean locally multiplicatively F-convex (NLMC) if there exists a base $\mathscr{B}$ of neighborhoods $U$ of 0 in $X$ such that for each $U \in \mathscr{B},(1) U$ is $F$-convex (i.e. if $\lambda$ and $\mu$ are scalars such that $|\lambda|,|\mu| \leqq 1$, then $\lambda U+\mu U \subset U$ ), and (2) $U U \subset U$.

DEFINITION 2. A seminorm $p$ on $X$ is nonarchimedean and multiplicative respectively if for all $x, y \in X(1) p(x+y) \leqq \max [p(x), p(y)]$ and (2) $p(x y) \leqq p(x) p(y)$.

Proposition 1. A topological algebra $X$ is an NLMC algebra iff the topology on $X$ is generated by a family $P$ of nonarchimedean multiplicative seminorms.

Proof. Given such a family $P$ generating the topology on $X$, the sets $\left\{x \mid p_{i}(x) \leqq \varepsilon, p_{1}, \cdots, p_{n} \in P, 0<\varepsilon \leqq 1\right\}$ form a base at 0 satisfying the condition of Definition 1.

Conversely, if $\mathscr{B}$ is a base at 0 satisfying the conditions of Definition 1, then, letting $p_{U}(x)=\inf \{|\mu| \mid x \in \mu U, \mu \in F\}$ the seminorms $\left(p_{U}\right)_{U \in \mathscr{B}}$ constitute the desired family $P$.

Proposition 2. If the valuation on $F$ is discrete and $X$ is an NLMC algebra, then there exists a family $P^{\prime}$ or nonarchimedean multiplicative seminorms generating the topology on $X$ such that $p^{\prime}(X) \subset|F|$ for each $p^{\prime} \in P^{\prime}$.

Proof. Let $P$ be a family of nonarchimedean multiplicative seminorms generating $X$ 's topology. For each $p \in P$ let $p^{\prime}(x)=\inf \{|\mu||| \mu \mid \geqq$ $p(x)\}$. Each such $p^{\prime}$ is clearly nonarchimedean and multiplicative. Moreover since $p(x) \leqq p^{\prime}(x) \leqq\left|\mu^{-1}\right| p(x)$ for any nonzero $\mu \in F$ such that $|\mu|<1$ and $|\mu|$ generates the value group of $F, P^{\prime}$ will also generate the topology on $X$.

DeFINITION 3. An NLMC algebra $X$ is discrete if there exists a family $P$ of nontrivial nonarchimedean multiplicative seminorms generating the topology on $X$ such that each $p$ in $P$ is discrete [the only limit point of $p(X)$ is 0$]$.

Proposition 3. A Hausdorff NLMC algebra $X$ is discrete iff $F$ is discretely valued.

Proof. Use Prop. 2.

If $X$ is a topological algebra over $C$, the complex numbers, then we can identify the nontrivial continuous homomorphisms of $X$ into $C$ with the closed maximal ideals in $X([9, \mathrm{p} .13])$. This is no longer 
true for noncomplex algebras, and we single out those algebras in which the $1-1$ correspondence still obtains for special attention.

DEFinition 4. A commutative Hausdorff NLMC algebra $X$ with identity $e$ is a Gelfand algebra if for every closed maximal ideal $M \subset X$ the factor algebra $X / M$ (with quotient topology) is topologically isomorphic to $F$.

Associated with the nontrivial nonarchimedean multiplicative seminorms $p$ generating the topology on an NLMC algebra $X$, are nonarchimedean normed algebras $X / N_{p}$ where $N_{p}$ is the ideal $p^{-1}(0)$ where $X / N_{p}$ is normed by taking $\left\|x+N_{p}\right\|=p(x)$. The completions $X_{p}$ of these normed algebras are referred to as factor algebras.

Proposition 4. If $X$ is a Gelfand algebra and $X / N_{p}$ is complete, then $X / N_{p}$ is a Gelfand algebra.

Proof. Let $\pi_{p}$ denote the continuous homomorphism $x \rightarrow x+N_{p}$ from $X$ onto $X / N_{p}$. We observe that if $M$ is a maximal ideal in the Banach algebra $X / N_{p}$, then $M$ is closed; thus $\pi_{p}^{-1}(M)$ is a closed maximal ideal in $X$ containing $N_{p}$. For any $x \in X$ there exists $\mu \in F$ such that $x-\mu e \in \pi_{p}^{-1}(M) \quad\left(X\right.$ is a Gelfand algebra), so that $\pi_{p}(x)-\mu \pi_{p}(e) \in M$ where $e$ is the identity of $X$. Thus $\left(X / N_{p}\right) / M$ is algebraically isomorphic to $F$. Since $M$ is closed, the factor structure is a one-dimensional Hausdorff topological vector space and is therefore topologically isomorphic to $F$.

Proposition 5. Let $P$ be a saturated family of seminorms generating the topology on the NLMC algebra $X$ and let $\left(X_{p}\right)_{p \in P}$ denote the associated factor algebras. If each $X_{p}$ is a Gelfand algebra, then $X$ is a Gelfand algebra.

Proof. Let $M$ be a closed maximal ideal in $X$. By [1, p. 466] there exists $p \in P$ such that $M \supset N_{p}$ and $\inf \{p(e-x) \mid x \in M\}>0$. Consequently $\pi_{p}(M)$ is a proper ideal in $X / N_{p}$ and $\pi_{p}(e)$ is not an adherence point of $\pi_{p}(M)$. Thus $\overline{\pi_{p}(M)}$ is a proper ideal in $X_{p}$ and is therefore contained in a closed maximal ideal $N \subset X_{p}$. Since $X_{p}$ is a Gelfand algebra, $N$ is the kernel of a continuous nontrivial homomorphism $f_{p}$ taking $X_{p}$ into $F$. Hence $f=f_{p} \pi_{p}$ is a continuous nontrivial homomorphism taking $X$ into $F$. It follows from elementary considerations that the kernel of $f$ is equal to $M$. Consequently $X / M$ is seen to be algebraically-hence topologically-isomorphic to $F$.

A result similar in spirit to this can be found in [2, p. 175]. We turn next to some examples. 
EXAMPLE 1. Let $F$ be a local field, let $T$ be a 0 -dimensional Hausdorff space and let $F(T)$ carry the topology of uniform convergence on compact sets. The topology on $F(T)$ is generated by the nonarchimedean multiplicative seminorms $p_{K}$ where $K$ is a compact subset of $T$ and for any $x \in F(T), p_{K}(x)=\sup _{t \in K}|x(t)|$. We may identify $F(T) / p_{K}^{-1}(0)$ with a subalgebra of $F(K)$. Moreover we may construct a'Stone-Cech' compactification $\beta_{F} T$ of $T$ as is done in [3, p. 243] utilizing the compact valuation ring $V$ of $F$ in place of the compact interval $[0,1]$. Since $V$ is Hausdorff and 0 -dimensional, $\beta_{F} T$ will be compact, Hausdorff and 0-dimensional. Thus the EllisTietze extension theorem ([4]) applies and any function continuous on $K$ may be extended to a function continuous on $\beta_{F} T$. It follows that $F(T) / p_{K}^{-1}(0)=F(K)$.

The continuous nontrivial homomorphisms of $F(K)$ into $F$ are in 1 - 1 correspondence with the points $t$ of $K$ ([11]) and using this result it can be shown $[9, p$. 31] that the points of $T$ generate the continuous nontrivial homomorphisms of $F(T)$ into $F . *$

Topological algebras $X$ for which all homomorphisms of $X$ into $F$ are continuous are called functionally continuous $[9, \mathrm{p} .51])$. What follows is an example of such an algebra.

EXAMPLE 2. Let $F$ be any complete nonarchimedean nontrivially valued field and $T$ a 0 -dimensional Hausdorff space. $F(T)$ carries the compact-open topology. A subalgebra $X$ of $F(T)$ is "closed under inverses" if when $x \in X$ and $x^{-1} \in F(T), x^{-1} \in X$. We apply Michael's proof [9, p. 54] and observe that if Conditions 1 and 2 below are satisfied, then the homomorphisms of $X$ are generated by the points of $T$ and therefore $X$ is functionally continuous.

1. For any $x_{1}, \cdots, x_{n} \in X$ such that $\bigcap_{i=1}^{n} x_{i}^{-1}(0)=\varnothing$, there exists $y_{1}, \cdots, y_{n} \in X$ such that $\Sigma x_{i} y_{i}=e$ where $e$ is the constant function $e(t)=1$ for all $t \in T$.

2. For some positive integer $m$ there exists $x_{1}, \cdots, x_{m} \in X$ such that for all $\mu_{1}, \cdots, \mu_{m} \in F, \cap\left(x_{i}-\mu_{i} e\right)^{-1}(0)$ is compact.

We note that if $X=F(T)$, then by the results of a sequel to this paper [16], it follows that $X$ satisfies statement 1 . If, in addition, there exists a bijection $x \in X$, then $X$ satisfies 2 . Hence if we take $T=F$ and let $T$ carry any 0 -dimensional Hausdorff topology finer than

* The result of Example 1 actually obtains if $F$ is any complete nonarchimedean nontrivially valued field as it can be shown in this case that a bounded continuous function defined on a compact subset $K$ of $T$ mapping into $F$ can be extended to a bounded continuous function mapping $T$ into $F$. The same comment applies to Example 1, parts (c), (d), and (e) of Sec. 2. 
the valuation topology on $F$, the nontrivial homomorphisms of the algebra $F(T)$ taking values in $F$ are generated by the points of $T$.

2. Function algebras over valued fields. In this section we discuss function algebras over valued fields. First we prove a version of a theorem of Kaplansky ([7, p. 173]) which is relevant to the material to follow; we include this proof because there seems to be an inconsistency in the use of "totally disconnected" in [7].

Lemma 1. (Kaplansky) Let $T$ be a topological space and let $F(T)$ be endowed with the topology of uniform convergence on compact sets. $I$ is a closed ideal in $F(T)$, iff there is some closed subset $H$ of $T$ such that $I=\{f \in F(T) \mid f(H)=\{0\}\} . \quad I$ is a closed maximal ideal in $F(T)$ iff there is some $t \in T$ such that $I=\{f \mid f(t)=0\}$.

Proof. Suppose $I$ is closed in $F(T)$ and let $H=\bigcap_{g \in I} g^{-1}(0)$. Letting $J(H)=\{f \mid f(H)=\{0\}\}$, we see that $I \subset J(H)$, and that $J(H)$ is a closed ideal. We show that if $f \in J(H)$, then $f \in I$.

Let $K$ be any compact subset of $T$. If $y \in K$, then as $I$ is an ideal, there exists $g_{y} \in I$ such that $g_{y}(y)=f(y)$. Since the clopen sets $\left\{U_{y} \mid y \in K\right\}$ where $U_{y}=\left\{x \in T|| f(x)-g_{y}(x) \mid<\varepsilon\right\}$ cover $K$ for any fixed $\varepsilon>0$, there exist $y_{1}, \cdots, y_{n}$ such that $K \subset \bigcup_{i=1}^{n} U_{y_{i}}$. Since the sets $U_{y_{i}}$ are clopen, we see that there exist pairwise disjoint clopen sets $W_{i}$ such that $K \subset \bigcup_{i=1}^{n} W_{i}$ where $W_{i} \subset U_{y_{i}}$ for each $i$. Letting $k_{A}$ denote the characteristic function of the set $A$, we see that if $h=$ $\sum_{i=1}^{n} g_{y_{i}} k_{W_{i}}$, then $h \in I$ and $\sup _{t \in K}|h(t)-f(t)|<\varepsilon$. As $\varepsilon>0$ can be made arbitrarily small, it follows that $f \in \bar{I}=I$..

In the proof to follow, "totally disconnected" is used as in [13, p. 380]: distinct points may be separated by clopen sets.

THEOREM 1. (Kaplansky) Let $S$ and $T$ be 0-dimensional Hausdorff spaces. Let $F(S)$ and $F(T)$ carry their compact-open topologies and suppose that $F(T)$ is topologically isomorphic to $F(S)$. Then $S$ and $T$ are homeomorphic.

Proof. Let $A$ be a topological isomorphism from $F(S)$ onto $F(T)$. If $K$ is a closed subset of $S$ and $J(K)$ denotes the ideal of functions that vanish on $K$, note that a mapping $A^{\prime}$ is defined by $A(J(\{s\}))=$ $J(\{t\})=J\left(\left\{A^{\prime}(s)\right\}\right)$ for some $t \in T$; i.e. $A^{\prime}: S \rightarrow T$ is such that $A^{\prime}(s)=t$, and is well-defined as $T$ is totally disconnected. Since $A$ is injective and $S$ is totally disconnected, then $A^{\prime}$ is injective as well. For any $t \in T, J(\{t\})=A(M)$ where $M$ is a closed maximal ideal in $F(S)$. 
Since $M=J(\{s\})$ for some $s \in S, A^{\prime}$ is seen to be surjective.

Clearly $\left(A^{\prime}\right)^{-1}=\left(A^{-1}\right)^{\prime}$ so to show that $A^{\prime}$ is a homeomorphism, it suffices to show that $A^{\prime}$ is a closed map. To this end, since $S$ is 0-dimensional, $K=\bigcap_{g \in J(K)} g^{-1}(0)$; since $J(K)=\bigcap_{s \in K} J(\{s\})$, it follows that $A(J(K))=J\left(A^{\prime}(K)\right)=\bigcap_{s \in K} J\left(\left\{A^{\prime}(s)\right\}\right)$. If $t \notin A^{\prime}(K)$, then $t=A^{\prime}(s)$ where $s \notin K$. Thus $J(K) \not \subset J(\{s\})$ and $J\left(A^{\prime}(K)\right) \not \subset J(\{t\})$. As $J\left(A^{\prime}(K)\right)=J\left(\overline{A^{\prime}(K)}\right) \not \subset J(\{t\})$, we see that $t \notin \overline{A^{\prime}(K)}$ and therefore $A^{\prime}(K)=$ $\overline{A^{\prime}(\bar{K})}$.

ExAmple 1. Let $T$ be a totally disconnected Hausdorff space and let $F(T)$ carry the compact-open topology. We note immediately that the set of evaluation maps constitutes a set of distinct continuous homomorphisms of $F(T)$ into $F$. Moreover properties (a)-(e) also hold.

(a) If $K$ is a compact subset of $T, p_{K}$ is as in Ex. 1 of Sec. 1, and $N_{K}=p_{K}^{-1}(0)$, then the completion of the normed algebra $F(T) / N_{K}$ is $F(K)$.

Proof. Since $T$ is totally disconnected, the characteristic functions in $F(T)$ separate the points of $T$. Thus the functions $\left.f\right|_{K}$ as $f$ runs through $F(T)$ separate points in $K$. The desired result now follows from an application of Kaplansky's Stone-Weierstrass theorem ([8] or [12] p. 161).

(b) With " $V^{*}$-algebra" as in [12, p. 148], if $T$ is locally compact, then $F(T)$ is the projective limit of $V^{*}$-algebras as in [9, p. 17].

Proof. The complete NLMC algebra $F(T)$ is the projective limit of the factor algebras $F(K)$ as $K$ runs through the compact subsets of $T$ and each $F(K)$ is a $V^{*}$-algebra.

(c) If $T$ is ultranormal and $F$ is a local field, then $F(T) / N_{K}=$ $F(K)$.

Proof. Use the Ellis-Tietze extension theorem of [4].

(d) If $T$ is 0 -dimensional and $F$ is a discretely valued field, then $F(T) / N_{K}=F(K)$ for any compact subset $K$ of $T$.

Proof. Apply a modification of the Ellis-Tietze extension theorem to functions $f \in F(K)$ and thereby extend $f$ continuously to a 'StoneCech' compactification $\beta_{H} T$ where $H$ is any local field. Where Ellis used local compactness of the field $F$, we use discreteness of the valuation on $F$, and compactness of $\beta_{H} T$.

(e) The points of $T$ constitute all continuous homomorphisms of $F(T)$ into $F$ when $F$ is discretely valued.

Proof. See Ex. 1 of Sec. 1 and use (d). 
3. Main results. Let $X$ be a NLMC algebra over a discretely valued $F$. Then, as in the classical case $([9, \mathrm{p} .33])$, if $X$ is the projective (dense inverse) limit of a family $\left(F\left(K_{n}\right)\right)$ of Gelfand $V^{*}$-algebras by mappings $\pi_{m n}: F\left(K_{n}\right) \rightarrow F\left(K_{n}\right), m>n$, where $\left(K_{n}\right)$ is a family of compact 0-dimensional Hausdorff spaces (it following that $K_{n}$ is homeomorphically embedded in $K_{m}$ ), then $X$ is topologically isomorphic to $F\left(\cup K_{n}\right)$ where* $F\left(\cup K_{n}\right)$ carries the compact-open topology. Moreover in this case $\cup K_{n}$ can and will be identified with the set of all nontrivial continuous homomorphisms of $X$ into $F$ and carries the weak topology generated by $\left(K_{n}\right)$.

Definition 1. Let $\mathscr{C}$ denote the nontrivial continuous homomorphisms of an MLHC algebra $X$ over $F$ into $F$, and let $/ C$ carry the weak-* topology. Let $F(\mathscr{C l})$ denote the algebra of continuous functions mapping $/ / C$ into $F$ with compact open topology and consider the map $\psi: X \rightarrow F(\mathscr{C l})$ where, for any $x \in X, \psi(x)(h)=h(x)$ for each $h \in \mathscr{l l}$. $X$ is called a full algebra if the homomorphism $\psi$ is an isomorphism of $X$ onto $F(\mathscr{C l})$.

In [9] E. A. Michael stated that he did not know whether or not $\psi$ was a topological isomorphism in the case where $X$ is a Fréchet full algebra. S. Warner proved that this was true in the classical case ([15, p. 269]). In this section we show that $\psi$ is a topological isomorphism if $F$ is a local field (Theorem 7). It then follows according to some results of van Tiel [14] that $X$ is the projective limit of a sequence $\left(F\left(K_{n}\right)\right)$ of Gelfand $V^{*}$-algebras where $K_{n}=V_{n}^{\circ} \cap \mathscr{L}\left(V_{n}^{\circ}\right.$ is the polar of a neighborhood $V_{n}$ of 0 in $X$ coming from a base of $F$-convex closed neighborhoods of 0 ). Thus we will have a partial converse of the result which was described in the opening paragraphs of this section. We also note that by Prop. 5 of Sec. $1, X$ is a Gelfand algebra under the hypothesis just mentioned.

In what follows $F$ is assumed to be discretely valued. In some cases it will also be assumed that $F$ is a local field so that certain standard results from the duality theory of topological vector spaces ([14]) may be used.

Definition 2. Let $F$ be discretely valued and let $\left(a_{\mu}\right)_{\mu \in H}$ be a system of distinct representatives of the cosets in the residue class field of $F$. We may assume that $H$ is totally ordered where $\mu_{0}$ corresponding to $a_{i_{0}}=0$ is the first element. Let $\pi \in F$ be such that $|\pi|<1$ and $|\pi|$ is a generator of the value group of $F$. If $a$ and $b$ are any two elements of $F$ there exist $\left(a_{\mu_{i}}\right)$ and $\left(a_{\lambda_{i}}\right)$ such that $a=$ $\sum_{i=N}^{\infty} a_{\mu_{i}} \pi^{i}$ and $b=\sum_{i=N}^{\infty} a_{\lambda_{i}} \pi^{i}$. We now define the supremum, $\sup (a, b)$,

* We may assume $K_{n} \subset K_{n+1}$ as there exist sets $K_{n}^{\prime}$ such that $\mathscr{C}=\cup K_{n}^{\prime}$ with $K_{n}^{\prime} \subset K_{n+1}^{\prime}$, and $K_{n}^{\prime}$ homeomorphic to $K_{n}$ for all $n$. 
of $a$ and $b$ as:

$$
\sup (a, b)=\left\{\begin{array}{l}
a \text { if }|a|>|b| \\
b \text { if }|b|>|a| \\
a \text { if } a=b \\
a \text { if }|a|=|b|, a_{\mu_{i}}=a_{\lambda_{i}} \text { for } i=N, \cdots, j-1 \text { and } \mu_{j}>\lambda_{j}
\end{array}\right.
$$

LeMma 1. Let $T$ be a topological space and let $f$ and $g$ be continuous functions mapping $T$ into $F$. Then the function defined at each $t \in T$ by $\sup (f(t), g(t))$ and denoted by $\sup (f, g)$ is continuous.

Proof. Suppose $\left(t_{s}\right)$ is a net in $T$ converging to $t$. We show that $\sup (f, g)\left(t_{s}\right)$ converges to $\sup (f, g)(t)$. Letting $f(t)=a$ and $g(t)=b$, we need only consider the last possibility for $\sup (a, b)$, the first three being trivial. Choose $\varepsilon>0$ such that $\varepsilon<|\pi|^{j}$. For $r$ such that $\left|f\left(t_{s}\right)-f(t)\right|<\varepsilon$ and $\left|g\left(t_{s}\right)-g(t)\right|<\varepsilon$ for $s \geqq r$, it follows that

$$
f\left(t_{s}\right)-f(t)=\sum_{i=M}^{\infty} a_{t_{i}}^{s} \pi^{i} \text { and } g\left(t_{s}\right)-g(t)=\sum_{i=M}^{\infty} a_{\lambda_{i}}^{s} \pi^{i}
$$

where $M>j$. We may also write

$$
f\left(t_{s}\right)=\sum_{1=N}^{j} a_{\mu_{i}} \pi^{i}+\sum_{i=j+1}^{\infty} a_{\mu_{i}}^{s} \pi^{i} \text { and } g\left(t_{s}\right)=\sum_{i=N}^{j} a_{\lambda_{i}} \pi^{i}+\sum_{i=j+1}^{\infty} a_{i_{i}}^{s} \pi^{i} .
$$

Thus, since $a_{i_{i}}=a_{\lambda_{i}}$ for $i=N, \cdots, j-1$, and $\mu_{j}>\lambda_{j}$, it follows that $\sup (f, g)\left(t_{s}\right)=f\left(t_{s}\right)$ for $s \geqq r$. Thus $\sup (f, g)\left(t_{s}\right)=f\left(t_{s}\right) \rightarrow f(t)=$ $\sup (f, g)(t)$.

LEMma 2. Let $F(T)$ denote the algebra of continuous functions mapping the 0-dimensional Hausdorff space $T$ into the discretely valued $F$, with compact-open topology. If $V$ is an $F$-barrel (closed absorbent $F$-convex set) in $F(T)$, then there is some $\delta>0$ such that $\sup _{t \in T}|\hat{f}(t)| \leqq \delta$ implies that $f \in V$.

Proof. Let $B$ be the sup-norm Banach space of all bounded functions from $T$ into $F$. We note that $V \cap B$ is an $F$-barrel in $B$. Since $B$ is $F$-barreled ([14, p. 268]) there is some $\delta>0$ such that $\sup _{t \in T}|f(t)| \leqq \delta$ which implies that $f \in V \cap B$.

Lemma 3. Let $V, F, T$ and $F(T)$ be as in Lemma 2, and suppose that for some compact subset $K$ of $T,\{f \mid f(K)=\{0\} \subset \subset V$. Then there is some $\mu>0$ such that whenever $\sup _{t \in K}|f(t)|<\mu$, then $f \in V$. Thus $V$ is a neighborhood of 0 in $F(T)$.

Proof. Let $a \in F$ and denote the function sending each $t \in T$ into $a$ by $a$. With $\delta$ as in Lemma 2 , choose $a \in F$ such that $0<|a| \leqq$ 
$\delta / 2$. Choosing an integer $n$ so that $\delta / n<|a|$, let $f \in F(T)$ be such that $\sup _{t \in K}|f(t)| \leqq \delta / n$. With $g=\sup (f, a)-a$, it follows that $g(t)=$ 0 for each $t$ in $K$. Thus $g \in V$. Since $|f(t)-g(t)| \leqq|a| \leqq \delta / 2$ for all $t \in T$, it follows that $f-g \in V$. Since $V$ is $F$-convex, $g+(f-g)=$ $f \in V$, and the proof is complete.

We continue towards nonarchimedean analogs of theorems of Nachbin (Theorem 3) and Warner (Theorem 7). First we consider a notion of support of a linear functional which serves to replace the classical notion used by Nachbin.

In Lemmas 4 and $5 F(T)$ again denotes the algebra of continuous functions from the 0-dimensional Hausdorff space $T$ into $F$ with compact-open topology and $\varphi$ denotes a member of the continuous dual $F(T)^{\prime}$ of $F(T)$. For any subset $S$ of $T, k_{S}$ denotes the characteristic function of $S$ taking values in $F$ and we note that $k_{S} \in F(T)$ iff $S$ is clopen. Let $\mathscr{S}$ denote the family of subsets $U$ of $T$ such that $U$ is clopen and $\varphi\left(f k_{U}\right)=0$ for all $f \in F(T)$.

LEMma 4. The family $\mathscr{S}$ has the following properties: (1) If $U$ is a clopen subset of $G \in \mathscr{S}$, then $U \in \mathscr{S}$; (2) $\mathscr{S}$ is a ring of sets.

Proof. To prove (1) we observe that $k_{U}=k_{G} k_{U}$. (2) follows readily from (1).

Definition 3. The support of $\varphi, F_{\varphi}$, is defined to be $C(\cup \mathscr{S})$.

We observe that since $\varphi$ is continuous there is some compact set $K \subset T$ and an integer $N$ such that if $f \in F(T)$, then $|\varphi(f)| \leqq N$ $\sup _{t \in K}|f(t)|$. Thus, if $f$ vanishes on $K$, then $\varphi(f)=0$.

THEOREM 1. In the same notation as above (1) $F_{\varphi} \subset K$ and therefore $F_{\varphi}$ is compact, (2) if $\varphi$ is nontrivial, then $F_{\varphi}$ is not empty, and (3) if $G \subset T$ is open and $G \cap F_{\varphi}$ is not empty, then there exists $f \in F(T)$ such that $f(C G)=\{0\}$ and $\varphi(f)=1$.

Proof. (1) If $G$ is a clopen subset of $C K$, then-since $k_{G}$ vanishes on $K-\varphi\left(f k_{G}\right)=0$ and $G \in \mathscr{S}$.

(2) If $F_{\varphi}$ is empty, $T=\cup \mathscr{S}$, and it follows that for some $U_{i} \in \mathscr{S}$, $K \subset \cup_{i=1}^{n} U_{i}=G$. Since $\mathscr{S}$ is a ring of sets, $G \in \mathscr{S}$ and since $C G$ is clopen and contained in $C K, \varphi(f)=\varphi\left(f k_{C G}\right)=0$ for all $f \in F(T)$. But then $\varphi$ is trivial.

(3) If $G \cap F_{\varphi} \neq \varnothing$, there is some $t \in G \cap F_{\varphi}$. Since $T$ is 0 dimensional, $t \in U \subset G$ where $U$ is clopen. Since $U \cap F_{\varphi} \neq \varnothing$, then $U \notin \mathscr{S}$ and there is some $g \in F(T)$ such that $\varphi\left(g k_{U}\right) \neq 0$. We of course may assume that $\varphi\left(g k_{U}\right)=1$. Letting $g k_{U}=f$, (3) is seen to be proved. 
In order to apply this notion of support to our version of Nachbin's theorem (Theorem 3) we require that $F_{\varphi}$ have the property that if $f$ vanishes on $F_{\varphi}, \varphi(f)=0$. We now develop a case where this is true and which makes the notion applicable to Theorem 3 as well as settling Michael's question in this setting (Theorem 7).

LEMma 5. Suppose that $\varphi(g)=0$ for any $g \in F(T)$ which vanishes on any clopen set $G$ containing $F_{\varphi}$. Then if $f$ vanishes on $F_{\varphi}$, $\varphi(f)=0$.

Proof. Suppose that $f \in F(T)$ vanishes on $F_{\varphi}$, and let $A_{n}=\{t \epsilon$ $T|| f(t) \mid<1 / n\}(n=1,2, \cdots)$. As $F_{\varphi} \subset A_{n}$ for any $n$ and $A_{n}$ is clopen $\varphi(f)=\varphi\left(f k_{A_{n}}\right)+\varphi\left(f\left(1-k_{A_{n}}\right)\right)$. By the hypothesis, since $f\left(1-k_{A_{n}}\right)$ vanishes on $A_{n}, \varphi(f)=\varphi\left(f k_{A_{n}}\right)$. Let $K$ be a compact subset of $T$ such that $|\varphi(f)| \leqq N \sup _{t \in K}|f(t)|$. Hence $|\varphi(f)|=\left|\varphi\left(f k_{A_{n}}\right)\right| \leqq N$ $\sup _{t \in K}\left|f k_{A_{n}}(t)\right|<N / n$. Since this is true for every $n, \varphi(f)=0$.

THEOREm 2. Let $T$ be a Lindelöf space. Then if $f$ vanishes on $F_{\varphi}, \varphi(f)=0$.

Proof. Let $G$ be a clopen subset containing $F_{\varphi}$. Since $C G$ is closed, $C G$ is Lindelöf. Since $C G \subset C F_{\varphi}=U \mathscr{S}$, there exist $U_{i} \in \mathscr{S}$ such that $C G \subset \bigcup_{i=1}^{\infty} U_{i}$. Since $\mathscr{S}$ is a ring, we may assume that the sets $U_{i}$ are pairwise disjoint. Since $C G \cap U_{i}=V_{i}$ is clopen and contained in $U_{i}$ then $V_{i} \in \mathscr{S}$. Thus $k_{C G}=\sum_{i=1}^{\infty} k_{V_{i}}$ in the topology of pointwise convergence on $F(T)$. We claim that the "pointwise convergence" of the preceding sentence may be replaced by "uniform convergence on compact sets."

To prove this last statement, let $L$ be a compact subset of $T$ and consider $L \cap C G$. As $L \cap C G$ is compact and contained in $\bigcup_{i=1}^{\infty} V_{i}$ there is some integer $N_{L}$ such that $n \geqq N_{L}$ implies that $L \cap C G$ is contained in $\bigcup_{i=1}^{n} V_{i}$. But $C G \subset \bigcup_{i=1}^{n} V_{i}$ so $L \cap C G=L \cap\left(\bigcup_{i=1}^{n} V_{i}\right)$. Thus for $n \geqq N_{L}, C G$ and $\bigcup_{i=1}^{n} V_{i}$ have the same points in common with $L$, and $\sup _{t \in L}\left|\left(k_{C G}-\sum_{i=1}^{n} k_{V_{i}}\right)(t)\right|=0$ for $n \geqq N_{L}$. Since $L$ was an arbitrary compact set, the series is seen to converge in the compact-open topology and $\varphi\left(f k_{C G}\right)=\sum_{i=1}^{n} \varphi\left(f k_{V_{i}}\right)=0$.

We now present a version of a theorem of Nachbin ([10, p. 472])

THeOREM 3. Let $F(T)$ denote the algebra of continuous functions mapping the 0-dimensional Hausdorff space $T$ into the discretely valued field $F$, with compact-open topology. Suppose that for each $\varphi \in F(T)^{\prime}$, $f$ vanishing on $F_{\varphi}$ implies $\varphi(f)=0$. Then $F(T)$ is $F$-barreled iff for every $E \subset T$ which is closed and not compact there is some $f \in F(T)$ 
which is unbounded on $E .^{*}$

Proof. Suppose that the condition holds and let $V$ be an $F$ barrel in $F(T)$. To show that $V$ is a neighborhood of 0 in $F(T)$ we begin by letting $K=\overline{\bigcup_{\varphi \in V^{0}} F_{\varphi}}$. If $K$ is not compact, let $f$ be unbounded on $K$ and consider the sets $A_{n}=\{t \in T|| f(t) \mid>n\}, n=1,2$, ... Each $A_{n}$ is clopen and $A_{n} \cap K \neq \varnothing$. Thus there is some $F_{\varphi_{n}} \subset$ $K$ such that $A_{n} \cap F_{\varphi_{n}} \neq \varnothing$. By Theorem 1 (3) there exists $f_{n} \in F(T)$ such that $f_{n}$ vanishes outside of $A_{n}$ and $\varphi_{n}\left(f_{n}\right)=1$. Since $\cap_{n=1}^{\infty} A_{n}=\varnothing$, the function $f=\sum_{n=1}^{\infty} a_{n} f_{n}$ is a continuous function for any choice of $a_{n} \in F$. As it is clear that $A_{m} \cap F_{\varphi_{n}}=\varnothing$ for all sufficiently large $m$, we may (by considering a subsequence) assume that $\varphi_{n}\left(f_{m}\right)=0$ for all $m>n$. By a proper choice of $a_{n}$ we see that $\left|\varphi_{n}(f)\right| \rightarrow \infty$ and as $\varphi_{n} \in V^{0}$, af cannot belong to $V^{00}=V$ no matter how small $|a|$ is. Thus we contradict the fact that $V$ is absorbent and $K$ must be compact. If $f$ vanishes on $K$, then $f$ vanishes on $F_{\varphi}$ for all $\phi \in V^{0}$. Thus $f \in$ $V^{00}=V$ so, by Lemma $3, V$ is a neighborhood of 0 .

To prove the converse, let $F(T)$ be $F$-barreled and $E$ be a closed noncompact subset of $T$. Let $V=\left\{f\left|\sup _{t \in E}\right| f(t) \mid \leqq \delta\right\}, \delta>0$, and let $K$ be a compact subset of $T$. As $E \cap C K \neq \varnothing$, using $\beta_{H} T$ as in Sec. 2 Ex. 1 (d) we may assert the existence of a sequence $\left(f_{N}\right)$ of functions which vanishes on $K$ but $\left|f_{N}\left(t_{N}\right)\right| \geqq N$ for any positive integer $N$ and some $t_{N} \in E$. Thus the $\operatorname{set}\left\{f\left|\sup _{t \in K}\right| f(t) \mid \leqq \varepsilon\right\} \not \subset V$ for any $\varepsilon>0$ and $V$ is not a neighborhood of 0 . It follows that $V$ is not absorbing and there exists $f \in F(T)$ which is unbounded on $E$.

CoROLlaRY. Let $T$ be a 0-dimenional Hausdorff Lindelöf space and $F$ a discretely valued field. Then $F(T)$ is $F$-barreled.

Proof. We refer to Theorem 2 and the construction of the function in the proof of Theorem 6 for the proof of the corollary.

THEOREM 4. Suppose the 0-dimensional Hausdorff space $T=\bigcup_{n=1}^{\infty} K_{n}$ where each $K_{n}$ is compact, $K_{n} \subset K_{n+1}$, and each compact subset of $T$ is contained in some $K_{n}$ (i.e. $T$ is hemicompact). Then denoting $T$ endowed with the weak topology ([3], p. 131) generated by the sets $\left(K_{n}\right)$ as $T_{w}, F(T)$ is dense in $F\left(T_{w}\right)$, each algebra carrying its compactopen topology.

Proof. Since the topology of $T_{w}$ is clearly stronger than that of $T$, $F(T) \subset F\left(T_{w}\right)$. We note that the topology of $T_{w}$ restricted to $K_{n}$ is

* In a sequel to this paper we show that Theorem 2 is true for any 0 -dimensional Hausdorff space $T$ and any complete nonarchimedean nontrivially valued field $F$. Thus Theorem 3 is true for all spaces $T$. We also show that the result of Theorem 3 holds of $F$ is spherically complete ([16]). 
equal to the topology $K_{n}$ inherits from $T$ and the compact subsets of $T_{w}$ lie in the sets $K_{n}$. Thus $F(T)$ is a topological subspace of $F\left(T_{w}\right)$. Using Sec. 2 Ex. 1 (d), $F(T) / N_{K}=F(K)$ for any compact set $K \subset T$ and it follows that $F(T)$ is dense in $F\left(T_{w}\right)$.

THEOREM 5. Let everything be as in the preceding theorem. If $F(T)$ is complete then $T=T_{w}$ iff $T_{w}$ is 0-dimensional.

Proof. If $F(T)$ is complete, then $F(T)=F\left(T_{w}\right)$. Since they are topologically isomorphic under the identity map by the proof of Theorem 4, if $T_{w}$ is 0 -dimensional, then $T=T_{w}$ by Theorem 1 of Sec. 2. We may also observe that the functions of $F(T)$ generate the topology of the space $T$ while those of $F\left(T_{W}\right)$ generate the topology of $T_{W}$. Thus as $F(T)=F\left(T_{W}\right)$, the topologies are equal.

THEOREM 6. Let $F(T)$ denote the algebra of continuous functions mapping the 0-dimensional Hausdorff space $T$ into the local field $F$ and suppose that $F(T)$ is a complete locally $F$-convex metric space with topology $\mathscr{T}$. If the homomorphisms determined by the points of $T$ are the $\mathscr{T}$-continuous homomorphisms, then $\mathscr{T}$ is the compactopen topology.

Proof. Let the set of evaluation maps determined by $T$ be denoted by $T^{*}$ and let $T^{*}$ carry the Gelfand topology (i.e. the weakest topology for $T^{*}$ with respect to which the maps $t \rightarrow x(t)$ of $T^{*}$ into $F$ are continuous for each $x \in F(T)$ ). Since $T$ is 0 -dimensional the Gelfand topology coincides with the original topology on $T$, i.e. $T$ and $T^{*}$ are homeomorphic. Since $(F(T), \mathscr{T})$ is $F$-barreled $([14$, p. 268]), the polar of any compact subset of $T^{*}$ is a neighborhood of 0 in $F(T)$. Thus, identifying $T$ and $T^{*}, \mathscr{T}$ is seen to be stronger than the compact-open topology on $F(T)$. If $F(T)$ with compact-open topology could be shown to be $F$-barreled, the closed graph theorem could be applied to complete the proof. To show that $F(T)$ is $F$-barreled, let $E$ be a closed noncompact subset of $T$. Since $F(T)$ is a Frechet space, $T^{*}$ is 0 -dimensional and Lindelöf and therefore $T$ is 0 -dimensional and Lindelöf. Thus $E$ is Lindelö and there exists a denumerable clopen cover $\left(U_{n}\right)$ from which no finite subcover can be extracted. We may assume the family $\left(U_{n}\right)$ to be pairwise disjoint. Since $C E$ is open in $T, C E=$ $\cup V_{\mu}$ where each $V_{\mu}$ is clopen so that $T=\left(\bigcup_{n=1}^{\infty} U_{n}\right) \cup\left(\bigcup_{n=1}^{\infty} V_{\mu_{n}}\right)$ where the $\left(V_{\mu_{n}}\right)$ may be assumed to be pairwise disjoint. Defining $H_{2 n}=$ $V_{\mu_{n}}, H_{2 n+1}=U_{n}$ and setting $L_{m}=H_{m}-\bigcup_{i=1}^{m-1} H_{i}$ then $T=\bigcup_{n=1}^{\infty} L_{n}$ where each $L_{n}$ is clopen and $\left(L_{n}\right)$ is pairwise disjoint. We note that $E$ must intersect infinitely many $L_{n}$ 's lest $E$ turn out to be covered by finitely many of the $U_{i}$. Now consider the function $f: T \rightarrow F$ 
defined by $f(t)=\sum_{i=1}^{\infty} a^{n} k_{L_{i}}(t)$ where $|a|>1$. We observe that $f$ is unbounded on $E$ and therefore $F(T)$ with compact-open topology is $F$-barreled.*

We now prove a nonarchimedean version of a theorem of Warner $([15$, p. 267]).

THEOREM 7. Let the set of nontrivial continuous homomorphisms on the Frechet full algebra $X$ be denoted by $\mathscr{L}$. Let $\mathscr{L}$ carry the weak-* (Gelfand) topology and $F(\mathscr{C l})$ the compact-open topology. Then $X$ is topologically isomorphic to $F(\mathscr{C l})$.

Proof. Carrying the topology of $X$ over to $F(\mathscr{C l})$ via the isomorphism $\psi$ (Def. 1 of Sec. 1) and noting that $\mathscr{C l}$ constitutes the set of nontrivial continuous homomorphisms of $F(\mathscr{C})$ into $F$, we see by the previous theorem that the proof is done.

For complex algebras, Warner ([15]) has proved that the "II" of Theorem 7 is a $k$-space ( $\mathscr{C}$ carries the weak topology generated by a sequence of compact sets). This question as well as an attempt to develop a substitute for concept of "Q-space" ([5, p. 271]) is investigated in subsequent papers ([16]).

\section{REFERENCES}

1. R. Arens, A generalization of normed rings, Pacific. J. Math., 2 (1952), 455-471.

2. — Dense inverse limit rings, Mich. J. Math., 5 (1958), 169-182.

3. J. Dugundji, Topology, Allyn and Bacon, Boston, 1966.

4. R. L. Ellis, A nonarchimedean analog of the Tietze-extension theorem, Indag. Math., 29 (1967), 332-333.

5. L. Gillman and M. Jerison, Rings of continuous functions, van Nostrand, Princeton, N.J., 1960.

6. B. E. Johnson, Continuity of homomorphisms of topological algebras, Proc. Camb. Phil. Soc., (1964), 171-172.

7. I. Kaplansky, Topological rings, Amer. J. Math., 69 (1947), 153-183.

8. - The Weierstrass theorem in fields with valuation, Proc. Amer. Math. Soc., 1 (1950), 356-357.

9. E. A. Michael, Locally multiplicatively-convex topological algebras, Memoirs Amer. Math. Soc., no. 11, 1952.

10. L. Nachbin, Topological vector spaces of continuous functions, Proc. Nat. Acad. Sci. U.S.A., 40 (1954), 471-474.

11. L. Narici, On nonarchimedean Banach algebras, Arch. Math. (Basel), 19 (1968), 428-435.

12. L. Narici, E. Beckenstein, and G. Bachman, Functional analysis and valuation theory, Marcel Dekker, New York, 1971.

13. M. H. Stone, Applications of the theory of Boolean rings to general topology, Trans. Amer. Math. Soc., 41 (1937), 375-481.

* As shown here, the hypothesis of Theorem 6 implies $T$ to be Lindelof. $T$ being Lindelöf however implies that all homomorphisms of $F(T)$ into $F$ are given by points of $T$ ([16]). 
14. J. Van Tiel, Espaces localement $K$-convexes, Indag. Math., 27 (1965), 249-289.

15. S. Warner, The topology of compact convergence on continuous function spaces, Duke Math. J., 25 (1958), 265-282.

16. G. Bachman and E. Beckenstein, L. Narici, Function Algebras over Valued Fields, I-IV Atti Accad. Naz. dei Lincei, Rend. Cl. di fis. mat., e nat., (1971-1972).

Received June 7, 1971.

ST. JOHN'S UNIVERSITY

Notre Dame College

STATEN IsLand, NEW YORK

AND

POLYTEChNIC INSTITUTE OF BROOKLYN

AND

ST. JOHN'S UNIVERSITY

JAMAICA, NEW YORK 11439 


\section{PACIFIC JOURNAL OF MATHEMATICS}

\section{EDITORS}

H. SAMELSON

Stanford University

Stanford, California 94305

C. R. Hовву

University of Washington

Seattle, Washington 98105
J. DugundJI

Department of Mathematics University of Southern California

Los Angeles, California 90007

RICHARD ARENS

University of California

Los Angeles, California 90024

\section{ASSOCIATE EDITORS}

E. F. BECKENBACH

B. H. NeumanN

F. WOLF

K. YoSHIDA

\section{SUPPORTING INSTITUTIONS}

UNIVERSITY OF BRITISH COLUMBIA

CALIFORNIA INSTITUTE OF TECHNOLOGY

UNIVERSITY OF CALIFORNIA

MONTANA STATE UNIVERSITY

UNIVERSITY OF NEVADA

NEW MEXICO STATE UNIVERSITY

OREGON STATE UNIVERSITY

UNIVERSITY OF OREGON

OSAKA UNIVERSITY
UNIVERSITY OF SOUTHERN CALIFORNIA

STANFORD UNIVERSITY

UNIVERSITY OF TOKYO

UNIVERSITY OF UTAH

WASHINGTON STATE UNIVERSITY

UNIVERSITY OF WASHINGTON

AMERICAN MATHEMATICAL SOCIETY

NAVAL WEAPONS CENTER

Printed in Japan by International Academic Printing Co., Ltd., Tokyo, Japan 


\section{Pacific Journal of Mathematics}

\section{Vol. 44, No. $1 \quad$ May, 1973}

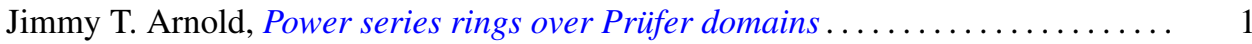

Maynard G. Arsove, On the behavior of Pincherle basis functions . . . . . . . . . 13

Jan William Auer, Fiber integration in smooth bundles ................. 33

George Bachman, Edward Beckenstein and Lawrence Narici, Function algebras

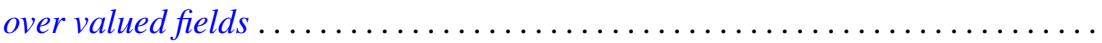

Gerald A. Beer, The index of convexity and the visibility function . . . . . . . . . . .

James Robert Boone, A note on mesocompact and sequentially mesocompact

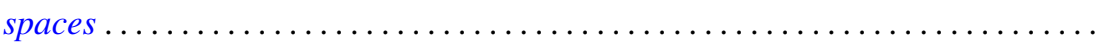

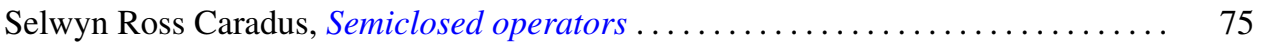

John H. E. Cohn, Two primary factor inequalities . . . . . . . . . . . . . . . 81

Mani Gagrat and Somashekhar Amrith Naimpally, Proximity approach to

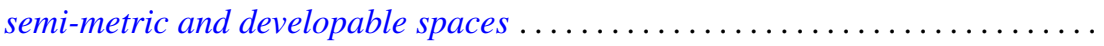

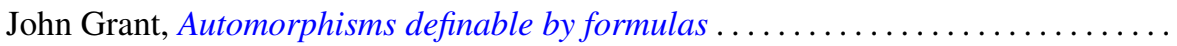

Walter Kurt Hayman, Differential inequalities and local valency ..............

Wolfgang H. Heil, Testing 3-manifolds for projective planes . . . . . . . . . . . . .

107

Melvin Hochster and Louis Jackson Ratliff, Jr., Five theorems on Macaulay

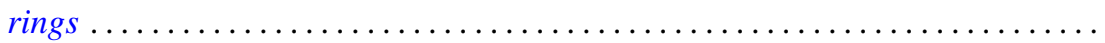

Thomas Benton Hoover, Operator algebras with reducing invariant subspaces ....

James Edgar Keesling, Topological groups whose underlying spaces are separable

Fréchet manifolds...

Frank Leroy Knowles, Idempotents in the boundary of a Lie group . .

191

George Edward Lang, The evaluation map and EHP sequences ...

201

Everette Lee May, Jr, Localizing the spectrum . . . . . . . . . . . .

211

Frank Belsley Miles, Existence of special $K$-sets in certain locally compact abelian groups.

Susan Montgomery, A generalization of a theorem of Jacobson. II . .

T. S. Motzkin and J. L. Walsh, Equilibrium of inverse-distance forces in

three-dimensions.

Arunava Mukherjea and Nicolas A. Tserpes, Invariant measures and the converse

of Haar's theorem on semitopological semigroups .

James Waring Noonan, On close-to-convex functions of order $\beta$

Donald Steven Passman, The Jacobian of a growth transformation

Dean Blackburn Priest, A mean Stieltjes type integral ........ .

Joe Bill Rhodes, Decomposition of semilattices with applications to topological

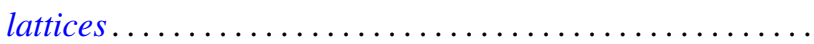

Claus M. Ringel, Socle conditions for $\mathrm{QF}-1$ rings ..........

Richard Rochberg, Linear maps of the disk algebra

Roy W. Ryden, Groups of arithmetic functions under Dirichlet convolution . .

Michael J. Sharpe, A class of operators on excessive functions

Erling Stormer, Automorphisms and equivalence in von Neumann algebras ..

Philip C. Tonne, Matrix representations for linear transformations on series

analytic in the unit disc. 